\title{
Sentence Formation in NLP Engine on the Basis of Indian Sign Language using Hand Gestures
}

\author{
Sumeet R. Agarwal \\ Dept. of Computer Engineering \\ Sinhgad College of Engineering \\ Vadgaon (Bk), Pune-41
}

\author{
Sagarkumar B. Agrawal \\ Dept. of Computer Engineering \\ Sinhgad College of Engineering \\ Vadgaon (Bk), Pune-41
}

\author{
Akhtar M. Latif \\ Dept. of Computer Engineering \\ Sinhgad College of Engineering \\ Vadgaon (Bk), Pune-41
}

\begin{abstract}
A person who is hearing impaired or mute is not able to compete or work with a normal person because of lack of proper communication between the two. This paper presents the development of an application which will enable a hassle free communication between physically disabled person and normal person. The application is responsible for the conversion of hand gestures into a meaningful sentence which can be read out by a normal person. In this approach, gestures based on Indian sign language are used. The major steps associated with the application are gesture recognition and natural language processing as most important module in the paper proposed. The current work can be used as an interface to communicate with speech impaired and normal person. Thus, it will bridge the gap between speech impaired and normal people by capturing sign processing it recognizing it $\&$ presenting output as meaningful sentence.
\end{abstract}

\section{General Terms}

Gesture Acquisition, Gesture Recognition, Natural Language Processing, Indian Sign Language, Sensor Glove.

\section{Abbreviations \\ ISL-Indian Sign Language, NLP- Natural Language Processing.}

\section{INTRODUCTION}

Machine Translation techniques started in seventeenth century and some remarkably successful natural language processing systems were developed late in nineteenth century in which a natural language system was restricted to be working in "block words" with limited vocabularies. But then there was a revolution in NLP by the development of algorithms for machine learning for processing of language. Today's research mainly focuses on semi-supervised and unsupervised learning algorithms. Natural language is the language which normal people use to communicate, and sign language is the language which is used by deaf and dumb people to communicate. Due to the rapid advancements in technology, people are trying to be a part of this technology. Nowadays cut throat competition in every field makes people with disabilities very difficult to stand in the race. The effort is to develop an application which will help deaf and dumb people communicate with a normal person. According to a survey, India composes of nearly 2.4 million deaf and dumb populations which approximately makes up $20 \%$ of the worlds total deaf and dumb population. For hassle-free communication between the normal person and deaf and dumb person, there is a need of an interpreter (Person who has the knowledge of sign language, as well as normal language).In the proposed idea, the application developed is more advanced in terms of communication between the deaf and dumb and normal person.In this approach, developing natural language processing engine is developed to construct meaningful sentences from sign language. The application is intended to capture hand gestures which are grammarless gestures and transform them into a meaningful sentence on the basis of the grammar designed. By the development of sentence formation application, the communication between the normal person and deaf and dumb people will be successful without any interpreter [10] [12].

The implementation of the proposed approach consists of modules like (1) Gesture Acquisition for Sign Language Input, (2) A Database Organization, (3)Natural Language Processing, for Sentence Formation.

The basic working model of the application is proposed below in Fig 1.

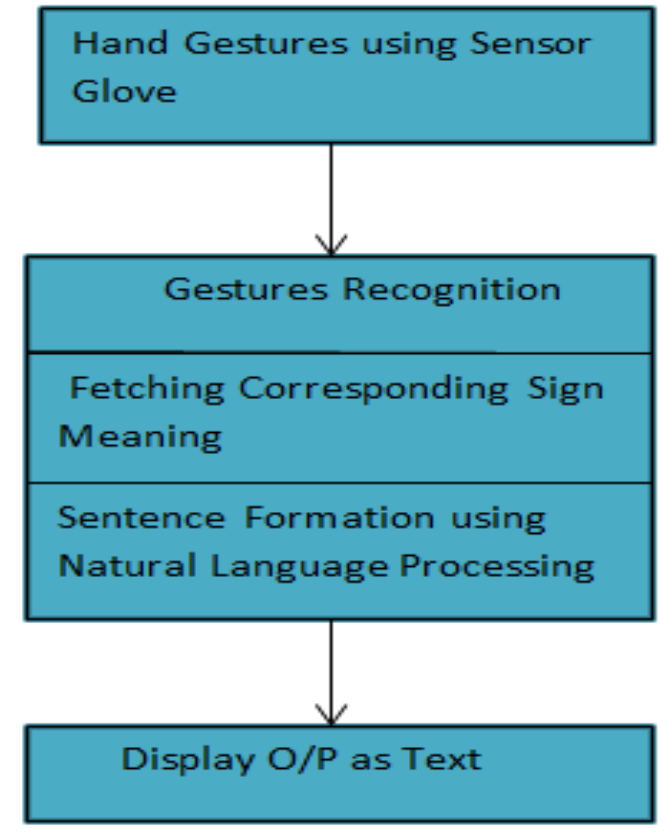

Fig 1: Module Structure

The development of this approach has many hurdles viz. (1)Total there are more than 2700 gestures in Indian Sign Language which contain maximum of dynamic gestures making it very difficult to recognize (2)Working out of sentences according to tenses is very difficult process.

\section{LITERATURE SURVEY}

Syed Atif Mehdi et.alproposes the use of sensor gloves for Sign Language Recognition. Sensor values coming from the sensor gloves is recognized with the help of artificial neural 
networks. The sensor values are further classified into 24 English alphabets and two punctuation symbols. The problem of dynamic gestures is discussed in the paper. This problem can be tackled with the help of gesture on the arm as sensor glove may not recognize dynamic gestures. The approach proposed in the paper was mainly to discuss the feasibility of recognizing sign languages with the help of sensor glove. The paper demonstrates that sensor glove can be used only for partial sign language recognition so to recognize full sign language more sensors can be used [1].

The Chung-Hsein Wu et.aldiscusses an approach for the formation of Chinese sentences for the people with hearing impairments from Taiwanese Sign Language. The people with language defects in sentence formation and grammatical correction are assisted by the proposed system [2].

Ualsher Tukeyev et.al explained the use of augmented attribute grammar for the conversion of a piece of text from one language to another. On the basis of context-free grammar, the formalization of the semantics of the language is provided by the attribute grammar. In the proposed methodology, demonstration of semantic analysis of text of natural language is applied with the help of attribute grammar [3].

Chandhana Surabhi Mproposes a novel idea of developing Natural language interfaces to communicate with machines. The application access to the user will be made easy by this approach [4].

Russel A. Kirsch et.alexplains the demonstration of an algorithm for sentence matching defined by simple grammar against a group of the set of simple pictures [5].

Finally the conclusion is that like other languages, ISL to English conversion is not just a word to word conversion. The identification of pronouns, prepositions and other grammatical parts of speech is a great challenge. The previous work on NLP and gesture recognition will be of great help for the development of the application. But there is always a need of innovative ideas for making something helping and creation of new software. So to develop the application an algorithm is to be developed.

\section{PROPOSED METHODOLOGY}

Deaf and dumb user and a normal user are two end users in the application.

The given Fig. 2 shows the workflow of the application. In the first step, deaf and dumb user will perform hand gestures on the basis of ISL using sensor glove. Sensor glove is of 5DT Company. The glove has seven sensors. Each of fingers and thumb has sensor on it. The other two are for rotation of hand and tilt of the hand. The bent of each sensor is measured with the help of value between 0 and 4095. 0 means the sensor is fully stretched, and 4095 means fully bent. These obtained values are matched to the database in order to obtain a unique word for each inputted gesture. These matched words are then passed to NLP engine where each word has a sign attribute on the basis of parts of speech of English grammar. These attributes are then used for construction of meaningful sentence on the basis of syntax of sentences in English grammar. The construction of sentences is accomplished by designing a grammar. The detailed explanation of the designed grammar is illustrated with the help of parse tree in next section (experimental setup). The obtained sentence is conveyed to a normal user in the text and speech form.[9] [11].

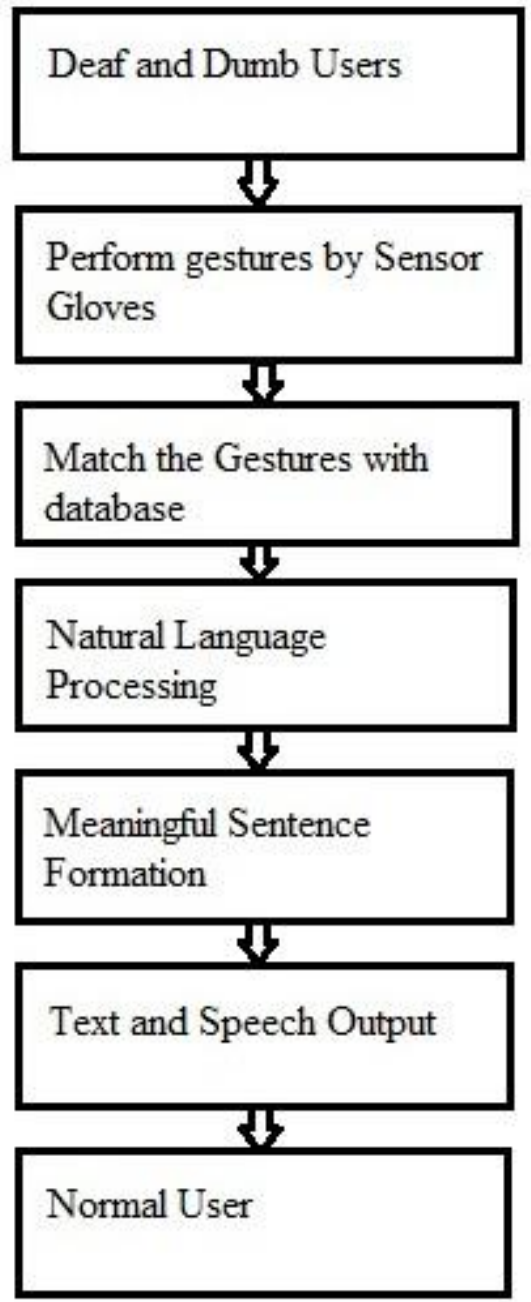

Fig 2: Workflow of the proposed methodology

\section{EXPERIMENTAL SETUP}

The designed grammar is represented as below:

$\mathrm{G}=\left\{\mathrm{V}, \sum, \mathrm{R}, \mathrm{S}\right\}$

where,

$\mathrm{V}=\{$ set of non-terminals $\}$

$=\{$

PRESENT_CONSONANT, PRESENT_VOWEL, PRESENT_PRONOUN,

PLURAL_PRESENT_PRONOUN,

CONSONANT_PRESENT_NOUN,

PLURAL_CONSONANT_PRESENT_NOUN,

CONSONANT_ADJECTIVE,

VOWEL_ADJECTIVE,

VOWEL_PRESENT_NOUN,

PAST_CONSONANT,

PAST_VOWEL,

PAST_PRONOUN, PLURAL_PAST_PRONOUN,

CONSONANT_PAST_NOUN,

PLURAL_CONSONANT_PAST_NOUN,

CONSONANT_ADJECTIVE,

VOWEL_ADJECTIVE, VOWEL_PAST_NOUN, FUTURE_CONSONANT, FUTURE_VOWEL, FUTURE_PRONOUN,

PLURAL_FUTURE_PRONOUN, CONSONANT FUTURE_NOUN, PLURAL_CONSONANT FUTURE_NOUN, CONSONANT_ADJECTIVE, 


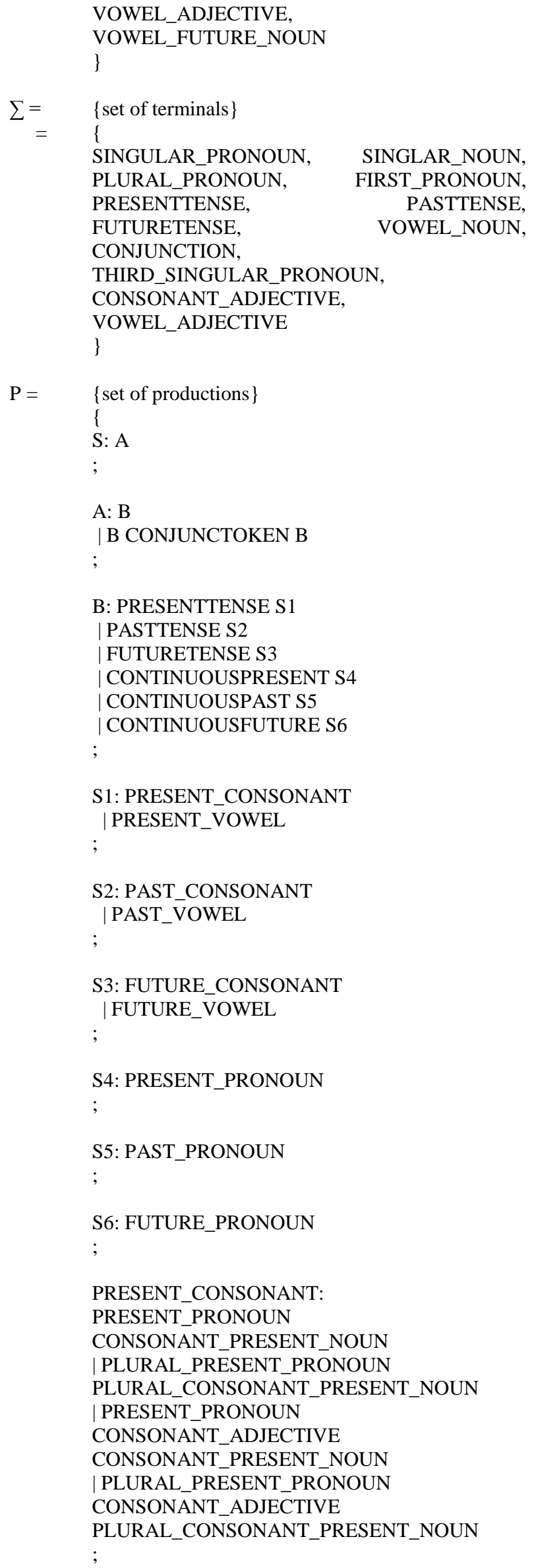

PRESENT_VOWEL: PRESENT_PRONOUN VOWEL_PRESENT_NOUN | PRESENT_PRONOUN VOWEL_ADJECTIVE VOWEL_PRESENT_NOUN ;

PRESENT_PRONOUN: SINGULAR_PRONOUN |FIRST_PRONOUN | THIRD_SINGULAR_PRONOUN ;

PLURAL_PRESENT_PRONOUN: PLURAL_PRONOUN 
CONSONANT_PAST_NOUN:

SINGULAR_NOUN| VOWEL_NOUN

;

PRESENT_CONSONANT_PAST_NOUN:

SINGULAR_NOUN | VOWEL_NOUN

;

CONSONANT_PAST_NOUN:

SINGULAR_NOUN

;

VOWEL_PAST_NOUN: VOWEL_NOUN

VOWEL_PAST_NOUN: SINGULAR_NOUN

| VOWEL_NOUN

;

FUTURE_CONSONANT: FUTURE_PRONOUN

CONSONANT_FUTURE_NOUN

| PRESENT_FUTURE_PRONOUN

PRESENT_CONSONANT_FUTURE_NOUN

| FUTURE_PRONOUN

CONSONANT_ADJECTIVE

CCONSONANT_FUTURE_NOUN

| PRESENT_FUTURE_PRONOUN

CONSONANT_ADJECTIVE

PRESENT_CONSONANT_FUTURE_NOUN

;

FUTURE VOWEL: FUTURE_PRONOUN

VOWEL_FUTURE_NOUN

|FUTURE_PRONOUN VOWEL_ADJECTIVE

VOWEL_FUTURE_NOUN
FUTURE_PRONOUN: SINGULAR_PRONOUN

| FIRST_PRONOUN

| THIRD_SINGULAR_PRONOUN

PRESENT_FUTURE_PRONOUN:

PLURAL_PRONOUN

;

PRESENT CONSONANT_FUTURE_NOUN: SINGULAR_NOUN | VOWEL_NOUN

;

CONSONANT_FUTURE_NOUN:

SINGULAR_NOUN

CONSONANT_FUTURE_NOUN:

SINGULAR_NOUN

| VOWEL_NOUN

;

VOWEL_FUTURE_NOUN: VOWEL_NOUN

;

VOWEL_FUTURE_NOUN: SINGULAR_NOUN

| VOWEL_NOUN

;

CONJUNCTOKEN: CONJUNCTION

;

$\mathrm{S}=$ Start Symbol

$S$

B

B CONJUNCTOKEN B

PRESENTTENSE S1 PASTTENSE S2 FUTURETENSE S3CONJUNCTION PRESENTTENSE S1 PASTTENSE S2FUTURETENSE S3

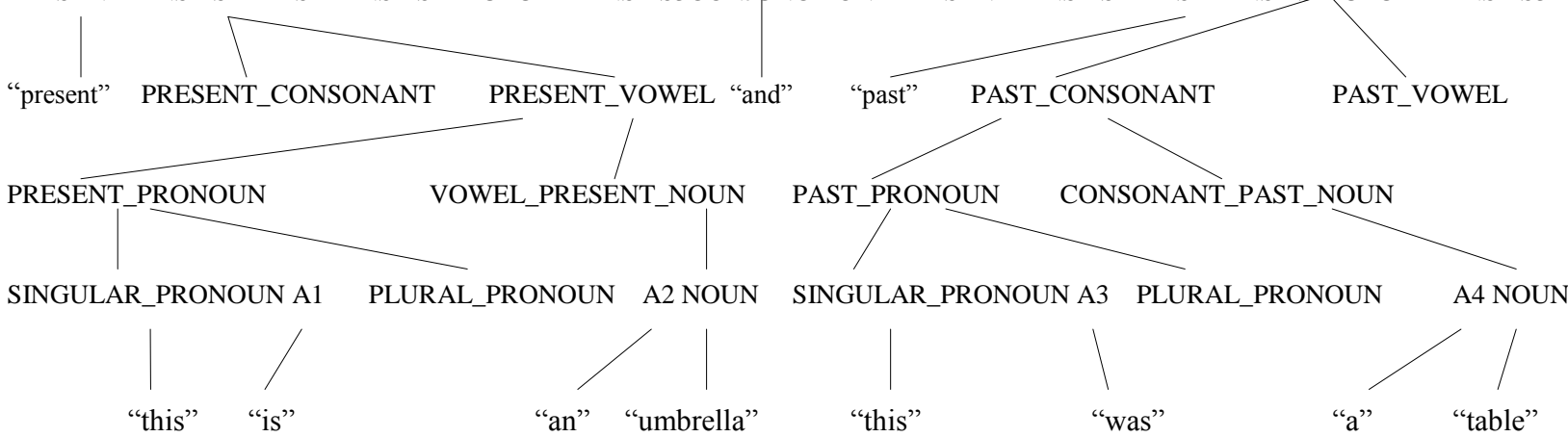

Fig 3: Parse Tree

The Fig 3 shown above explains the LALR (Look Ahead LR) bottom up parsing. The inputs to the parser are the parts of speech of English language viz. Pronoun, noun, adjective, verb, conjunction, etc. The Indian Sign Language does not consist of gestures for verbs like is, am, are, was, were and for articles. Therefore to construct a meaningful sentence from sign gestures there is the need of a language processor which willinsert verbs and articles at appropriate places. The approach can be explained with the help of parse tree given in Fig 3. 
The example in Fig 3 inputs to parser are "present", "this", "umbrella"," and", "past", "this" and "table". Here present and past indicate the simple tenses. The expected output from the above input is "this is an umbrella and this was a table". The categorization of input is done according to the basic rules of English grammar. "this" is categorized as singular pronoun and "umbrella", "table" as noun and "and" as conjunction. In the input the first tense is specified as "present" and "this"being a singular pronoun, the verb "is" is appropriate. Similarly, same logic can be applied for all other simple tenses. As "umbrella" is a noun and starting with a vowel, "an" is appended before it. In this way, two simple sentences can be constructed, and compound sentence can be formed by using conjunctions such as "and", "or", "but", etc. Thus, simple sentences and compound sentences can be constructed using the specified grammar.

\section{RESULT AND DISCUSSION}

The grammar discussed above is a context-free grammar. In the initial stages of development of the application in version 1, the grammar comprised of Non-Deterministic Finite Automata with $€$ moves ( $€$-NFA). For better results and hence better accuracy, the conversion of $€$-NFA to Deterministic Finite Automata (DFA) is done. Thus, in version 2 of the application, better results were obtained because; DFA consists of each state as a unique state. Therefore, the DFA developed does not contain any ambiguity. Moreover, the conversion of $€$-NFA to DFA led to the removal of ambiguity from the grammar and expanded its scope to a great extent.

It is very difficult to handle all types of sentences and all types of tenses. In the version 1 of the application, the tense was not an input parameter while performing gestures. Thus the sentence generated was found to be $33.33 \%$ correct. This can be explained with the help of following example.

Table 1. Evaluation of version 1 of application

\begin{tabular}{|l|l|l|}
\hline Gestures Performed & Outcome & Accuracy \\
\hline \multirow{3}{*}{ "this" "table" } & This was a table & $33.33 \%$ \\
\cline { 2 - 3 } & This is a table & $33.33 \%$ \\
\cline { 2 - 3 } & This will be a table & $33.33 \%$ \\
\hline
\end{tabular}

In version 2 , the accuracy of outcome was increased by prompting user to input the tense in which the sentence has to be constructed. Therefore, the user will specify the tense and sentence will be evaluated accordingly. This is demonstrated with the help of following example.

Table 2. Evaluation of version 2 of application

\begin{tabular}{|l|l|l|}
\hline Gesture Performed & Outcome & Accuracy \\
\hline "past" "this" "table" & This was a table & $100 \%$ \\
\hline "present" "this" "table" & This is a table & $100 \%$ \\
\hline "future" "this" "table" & This will be a table & $100 \%$ \\
\hline
\end{tabular}

In the application, only simple and continuous tenses are tackled for simple and compound sentences. The grammar developed generates only those sentences which are structurally correct as per the grammar rules. Hence in some cases meaningful sentence may not be constructed.

\section{FUTURE SCOPE}

All body gestures can be used to construct more types of sentences. The grammar can be improved further to construct all types of sentences. Moreover, the application can be designed to work in reverse manner.

\section{CONCLUSION}

The application has great significance in India because of less development in this field as compared to India's population. In this approach, the results which are obtained are very promising. The whole set of modules developed in the application will definitely help in narrowing the gap between the deaf and dumb person and normal person. The gesture acquisition and formation of meaningful sentences from the detected gestures work successfully. The application works as an interpreter for the communication between deaf and dumb person and normal person. The application can be further used for other future projects. It is a platform for deaf and dumb people to stand in the race with normal people.

\section{REFERENCES}

[1] Syed Atif Mehdi, Yasir Niaz Khan, "Sign Language Recognition Using Sensor Glove", in $9^{\text {th }}$ International Conference on Neural Information Processing Vol.5,2002.

[2] Chung-Hsein Wu, IEEE, Yu-Hsein Chiu and Chi Shiang Guo, "Text Generation From Taiwanese Sign Language Using PST-Based Language Model for Augmentative Communication", in Vol 12, No. 4, December 2004.

[3] Ualsher Tukeyev and Diana Rakhimova, "Augmented Attribute Grammar in Meaning of Natural Language Sentences", SCIS-ISIS 2012, Kobe, Japan, November 20-24, 2012.

[4] Chandhana Surabhi M, "Natural Language Processing Future", International Conference on Optical imaging sensor and security, Coimbatore, Tamil Nadu, India, July $2-3,2013$

[5] Russel A. Kirsch, "Computer Interpretation of English Text and Picture Patterns", IEEE Transactions on Electronic Computers.

[6] Ananya Choudhury, Anjan Kumar Talukdarand Kandarpa Kumar Sarma, "A Conditional Random Field based Indian Sign Language Recognition System under Complex Background", Fourth International Conference on Communication Systems and Network Technologies, 2014.

[7] Arti Thorat, Varsha Satpute, Arati Nehe, Tejashri Atre Yogesh R Ngargoje, "Indian Sign Language Recognition System for Deaf People", International Journal of Advanced Research in Computer and Communication Engineering Vol. 3, Issue 3, March 2014.

[8] P. Subha Rajam, Dr. G. Balakrishnan, "Real Time Indian Sign Language Recognition System to aid Deaf-dumb People" in IEEE Conference, 2011.

[9] Futane P.R,Dharaskar R.V, "Hasta Mudra: An interpretation of Indian sign hand gestures", 3rd International Conference on Electronics Computer Technology (ICECT), 8-10 April, 2011.

[10] "Prevention of Deafness", by Dr. Raj Desai,http://www.projectdeafindia.org.

[11] "5DT Data Glove Ultra", Fifth Dimension Technologies, http://www.5dt.com/products/pdataglove5u.html.

[12] "History of Machine Translation", from Wikipedia http://www.wikipedia.org/wiki/History_of_machine_tran slation. 